\title{
Red chillies tackle tumours
}

Red chilli peppers have been used for thousands of years as food additives and in traditional medicine. Their principal active component is capsaicin, which is a powerful stimulant of afferent nerve fibres. Intriguingly, dendritic cells - key orchestrators of the immune response - also express receptors for capsaicin and with this in mind Beltran et al. investigated the effects of capsaicin on the immune system. Their study, reported in the Journal of Immunology, shows that intratumoural administration of capsaicin results in not only the regression of the injected tumour, but also in growth inhibition of other uninjected tumours.

The capsaicin receptor vanilloid receptor 1 (VR1) is mainly expressed on sensory neurons where it conveys pain sensation. However, capsaicin has also been shown to have immunostimulatory properties that affect lymphocyte proliferation and serum antibody levels. The engagement of VR1 on dendritic cells leads to their maturation, resulting in an upregulation of antigen-presenting and co-stimulatory molecules on their surface, and can induce the migration of dendritic cells to draining lymph nodes.

To investigate whether this adjuvant ability of capsaicin can have an application in tumour therapy, the authors challenged mice with the aggressive fibrosarcoma Meth A. Indeed, the injection of capsaicin into palpable tumours of various sizes at 5 or 10 days after challenge, and again
5 days later, resulted in either complete regression or slowed growth of the injected tumour. In vitro experiments demonstrated that capsaicin can directly induce apoptosis in Meth A cells, and also in the fibrosarcoma cell line CMS5, but not in normal fibroblasts, in a time- and dose-dependent manner. Further in vivo experiments demonstrated that capsaicin treatment not only leads to regression of the injected tumour, but also of untreated tumours of the same type - indicating that antigen-specific immune-mediated mechanisms are involved in tumour-cell destruction.

To further investigate the role of capsaicin on the immune system, the authors challenged mice with a colon cancer cell line, which has a well-defined cytotoxic T-cell (CTL) epitope. After capsaicin treatment, antitumour CTL activity was significantly enhanced and tumours regressed. However, tumour growth was not affected in mice that lack functional $\mathrm{T}$ cells.

There may be two possible mechanisms for capsaicin's immunological activities, which possibly act synergistically. One is that capsaicin-mediated apoptosis of tumour cells could lead to the initiation of an immune response. As apoptosis is normally thought to be immunologically quiescent, the authors suggest that capsaicininduced apoptosis is immunologically active - this is in accordance with recent reports for other apoptotic agents, such as $\gamma$-irradiation. The second mechanism could be that direct dendritic-cell activation by capsaicin can lead to priming of a more efficient tumour-specific CTL response. The potency of capsaicin as an immuno-active ligand together with its cytotoxic effects and the induction of a specific immune response could make it an attractive candidate for cancer immunotherapy.

$$
\text { Alexandra Flemming }
$$

ORIGINAL RESEARCH PAPER Beltran, J. et al. Immunotherapy of tumors with neuroimmune ligand capsaicin.J. Immunol. 178, 3260-3264 (2007) FURTHER READING Basu, S. \& Srivastava, P. Immunological role of neuronal receptor vanilloid receptor 1 expressed on dendritic cells. Proc. Nat Acad. Sci. USA 102, 5120-5125 (2005) 